\title{
Nanoscale Detection of a Single Fundamental Charge in Ambient Conditions Using the $\mathrm{NV}^{-}$Center in Diamond
}

\author{
Florian Dolde, ${ }^{1, *}$ Marcus W. Doherty,${ }^{2}$ Julia Michl, ${ }^{1}$ Ingmar Jakobi, ${ }^{1}$ Boris Naydenov, ${ }^{3}$ Sebastien Pezzagna, \\ Jan Meijer, ${ }^{4}$ Philipp Neumann, ${ }^{1}$ Fedor Jelezko, ${ }^{3}$ Neil B. Manson, ${ }^{2}$ and Jörg Wrachtrup ${ }^{1}$ \\ ${ }^{1}$ 3. Physikalisches Institut, Research Center SCoPE and IQST, Universität Stuttgart, \\ Pfaffenwaldring 57. D-70550 Stuttgart, Germany \\ ${ }^{2}$ Laser Physics Centre, Research School of Physics and Engineering, Australian National University, \\ Australian Capital Territory 0200, Australia \\ ${ }^{3}$ Institut für Quantenoptik and IQST, Universität Ulm, Ulm D-89073, Germany \\ ${ }^{4}$ Physikalisches Institut, Universität Leipzig, 04103 Leipzig, Germany
}

(Received 11 October 2013; published 3 March 2014)

\begin{abstract}
Single charge nanoscale detection in ambient conditions is a current frontier in metrology that has diverse interdisciplinary applications. Here, such single charge detection is demonstrated using two nitrogen-vacancy (NV) centers in diamond. One NV center is employed as a sensitive electrometer to detect the change in electric field created by the displacement of a single electron resulting from the optical switching of the other $\mathrm{NV}$ center between its neutral $\left(\mathrm{NV}^{0}\right)$ and negative $\left(\mathrm{NV}^{-}\right)$charge states. As a consequence, our measurements also provide direct insight into the charge dynamics inside the material.
\end{abstract}

PACS numbers: 76.70.Hb, 07.50.Ls, 61.72.jn, 84.37.+q

Single charge nanoscale detectors that operate under ambient conditions have diverse interdisciplinary applications as probes of physical phenomena [1-3], components of quantum and nanodevices [4,5], and as highperformance sensors of chemical and biological species $[6,7]$. The detection of elementary charges is a longstanding endeavor, with a suite of low temperature or pressure techniques available, including single-electron transistors [1,2,4], scanning probe microscopy [3,8,9], electric field-sensitive atomic force microscopy [10], electromechanical resonators $[11,12]$, and nanowire fieldeffect transistors [13]. Yet, few techniques are available that operate under both ambient temperature and pressure and can detect small numbers of elementary charges [14]. Recently, the atomic-sized negatively charged nitrogenvacancy $\left(\mathrm{NV}^{-}\right)$center in diamond was demonstrated in ambient conditions to be a high-sensitivity electrometer with potential nanoscale applications [15]. Indeed, it was projected that the $\mathrm{NV}^{-}$center could be used to detect the electric field of a single electron at a distance of $\sim 150 \mathrm{~nm}$ within $1 \mathrm{~s}$ of averaging. Here, we demonstrate a vital first advance in single charge detection using the $\mathrm{NV}^{-}$center by sensing the presence of a single electron at a distance of $25 \pm 2 \mathrm{~nm}$. In fact, the single electron to be detected originates from a proximal second NV center. This NV pair is well characterized and enables deterministic control of a local charge in an otherwise clean test environment. Nanoscale scanning-probe electrometry is feasible by scanning a suitable NV diamond probe over a sample of interest as demonstrated for magnetometry [16,17].

Beyond electrometry, the $\mathrm{NV}^{-}$center has a range of impressive applications including, high-sensitivity nanomagnetometry [18-20] and thermometry [21-23], quantum information science [24,25], and bioimaging [26]. Each of these applications exploit some combination of the center's remarkable properties under ambient and extreme conditions $[27,28]$ : strong fluorescence that enables the detection of atom-sized single centers [29], long-lived ground state electron spin coherence, and optical spin polarization or readout [30]. More specifically, the NV center is a molecular point defect in diamond [see Fig. 1(b)]. Negatively and neutrally charged $\mathrm{NV}$ centers $\left(\mathrm{NV}^{-}\right.$and $\left.\mathrm{NV}^{0}\right)$ can be optically distinguished by their ZPLs (zero phonon line) at 637 and $575 \mathrm{~nm}$, respectively, which are each accompanied by broad $(\sim 100 \mathrm{~nm})$ phonon sidebands [30]. Additionally, $\mathrm{NV}^{-}$exhibits a ground state spin triplet with a zero-field splitting between its $m_{s}=0$ and \pm 1 spin sublevels of $D \sim 2.87 \mathrm{GHz}$ at room temperature. The spin triplet sublevel $m_{S}=0$ of the ground state can be initialized and read out via optical excitation to the excited state spin triplet. Spin-orbit and spin-spin mixing of the triplet levels makes the ground state spin resonances susceptible to electric and crystal strain fields [15,31,32]. The long-lived ground state spin coherence thus enables small electric field shifts of the spin resonances to be sensitively detected using optically detected magnetic resonance (ODMR) techniques.

ODMR electrometry may be further introduced via the $\mathrm{NV}^{-}$ground state spin Hamiltonian $[31,33]$

$$
\begin{aligned}
H= & \left(D+k_{\|} E_{z}\right)\left(S_{z}^{2}-2 / 3\right)+\frac{g_{e} \mu_{B}}{h} \vec{S} \cdot \vec{B} \\
& -k_{\perp} E_{x}\left(S_{x}^{2}-S_{y}^{2}\right)+k_{\perp} E_{y}\left(S_{x} S_{y}+S_{y} S_{x}\right),
\end{aligned}
$$

where $\vec{S}$ are the $S=1$ dimensionless electron spin operators, $\mu_{B}$ is the Bohr magneton, $g_{e} \sim 2.003$ is the electron 

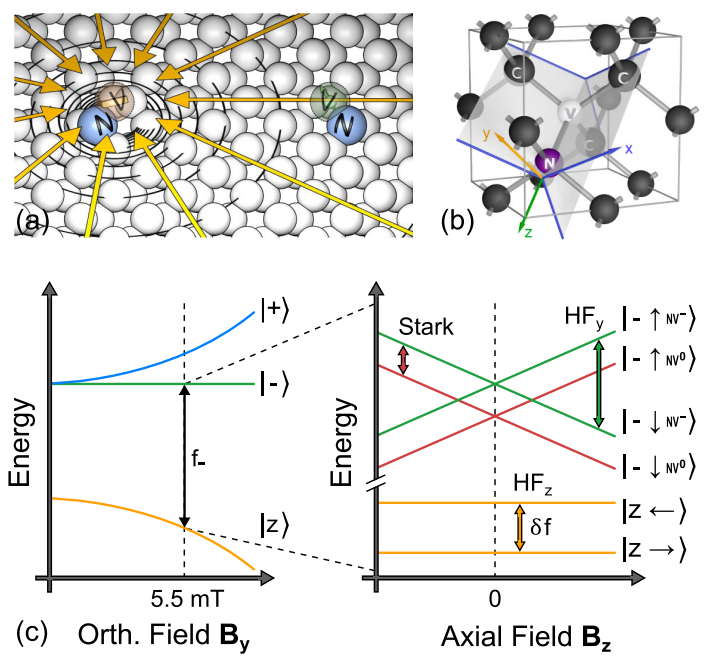

FIG. 1 (color online). (a) Schematic of the NV centers under investigation. On the right, NV $A$ is depicted as the electrometer $\mathrm{NV}$, and on the left NV $B$ is responsible for the electric field. (b) Diamond unit cell containing an NV center. The latter is a $C_{3 v}$ point defect (see mirror planes) consisting of a substitutional nitrogen $(\mathrm{N})$ - carbon vacancy $(\mathrm{V})$ pair orientated along the $\langle 111\rangle$ crystal $(z)$ axis. The negative charge state $\left(\mathrm{NV}^{-}\right)$is formed from the neutral $\mathrm{NV}^{0}$ when the center traps an excess electron. The local Cartesian coordinate axes $x, y$ and $z$ (blue, orange, green) are shown. (c) Left: Electron spin levels affected by a transverse magnetic field $B_{\perp}$ (here, $B_{y}$ ) of varying strength. Right: Effects on energy levels of hyperfine (HF) coupled electron spin nuclear spin pair due to additionally applied electric and longitudinal magnetic fields (Stark and $B_{z}$, respectively). See text for discussion.

$g$ factor [34,35], $h$ is the Planck constant, $\vec{B}$ and $\vec{E}$ are the magnetic and electric fields, respectively, $k_{\|}=$ $0.035(2) \mathrm{kHz} \mathrm{m} / \mathrm{V}$ and $k_{\perp}=1.7(3) \mathrm{kHz} \mathrm{m} / \mathrm{V}$ are the electric susceptibility parameters [32], and the spin coordinate system is defined such that the $z$ coordinate axis coincides with the center's trigonal symmetry axis and the $x$ axis is contained in one of the center's mirror planes [see Fig. 1(b)]. Given the orders of magnitude difference between $k_{\|}$and $k_{\perp}$, the electron spin is most sensitive to electric fields that are transverse to the center's trigonal axis (i.e., within the $x-y$ plane). Sensitivity is enhanced by tailoring the electron spin eigenbasis $\{|z\rangle,|-\rangle,|+\rangle\}$ using a transverse magnetic field, such that the $|0\rangle \leftrightarrow| \pm\rangle$ electron spin resonance frequencies $f_{ \pm}$are linearly susceptible to both axial and transverse electric field components $[15,31,33]$

$$
f_{ \pm} \approx f_{ \pm}(0)+k_{\|} E_{z} \mp k_{\perp} E_{\perp} \cos \left(2 \phi_{B}+\phi_{E}\right),
$$

where $\quad \tan \phi_{B}=B_{y} / B_{x}, \quad \tan \phi_{E}=E_{y} / E_{x}, \quad B_{\perp}=$ $\sqrt{B_{x}^{2}+B_{y}^{2}}, E_{\perp}=\sqrt{E_{x}^{2}+E_{y}^{2}}$, and $f_{ \pm}(0)$ are the resonance frequencies in the absence of an electric field, which depend on $B_{\perp}$, but not $\phi_{B}$. The bare electric field shift is given by $\Delta f_{ \pm}=f_{ \pm}-f_{ \pm}(0)$. As demonstrated in Ref. [15], the effects of the transverse orientations of the electric and magnetic fields on the spin resonances are coupled by the final term in Eq. (2), which can be observed by rotating the magnetic field in the transverse plane. Please note that the tailored eigenstates $\{|+\rangle,|-\rangle,|z\rangle\}$ are not susceptible to magnetic fields at first order, allowing for longer coherence times [15] and reducing spin-spin coupling strengths of the NV pair.

The neutral charge state $\mathrm{NV}^{0}$ is characterized by an optical ZPL at $\sim 2.156 \mathrm{eV}(575 \mathrm{~nm})$ accompanied by phonon sidebands. In the absence of light, the equilibrium $\mathrm{NV}$ charge state is determined by the local distribution of electron donors and acceptors [30]. Alternatively, the equilibrium NV charge state may be controlled via gate voltages applied to the diamond [36,37]. Different photoconversion processes enable controlled optical switching of the NV charge state [38-42]. Under red $(637-575 \mathrm{~nm})$ excitation, $\mathrm{NV}^{-}$is selectively excited and, subsequently, an electron is transferred to the conduction band, converting the center to $\mathrm{NV}^{0}$. Under green $(490-575 \mathrm{~nm})$ excitation, both charge states are excited and photoconversion occurs in both directions. However, the negative charge state is the dominant one with respect to occurrence and fluorescence intensity in the detected spectral range.

Improved understanding of the charge dynamics of the $\mathrm{NV}$ center [35,38-47] is particularly important to the performance of $\mathrm{NV}^{-}$in its various applications. However, there has not yet been a direct observation of the excess electron whose presence or absence determines the center's charge state. Here, we perform such a direct observation that unequivocally confirms the current charge state assignments and also provides insight into the microscopic behavior of the excess electron.

In our experiments, we employed a well-characterized pair of implanted ${ }^{15} \mathrm{NV}^{-}$centers that were oriented along different [111] directions in type IIa diamond and whose positions have been previously established using superresolution microscopy $[24,48]$. One center (NV A) was employed as an electrometer to detect the change in electric field created by the displacement of the excess electron of the other center ( $\mathrm{NV} B$ ) when it is deliberately optically switched from $\mathrm{NV}^{-}$to $\mathrm{NV}^{0}$. By selectively addressing the ODMR transitions of NV $A$, we were able to perform electrometry with NV A using polarized green $(532 \mathrm{~nm})$ spin polarization and readout laser pulses (timed with fluorescence detection) and optically switch the charge state of NV $B$ using polarized red $(638 \mathrm{~nm})$ pump laser pulses [33]. The length of the red pulse $\tau_{\text {pump }}$ controlled the probability that NV $B$ was in a given charge state during the ODMR measurement [33]. Similar to Ref. [15], the ODMR electrometry utilized a static transverse bias magnetic field with magnitude $B_{\perp} \approx 5.5 \mathrm{mT}$, a smaller auxiliary magnetic field $\delta \vec{B}$ that tuned the net magnetic field [see Fig. 3(a)], and a Ramsey-type microwave pulse sequence with free spin evolution time $\tau$. The transverse bias magnetic field split the $|z\rangle \leftrightarrow| \pm\rangle$ electron spin resonances and the microwave pulses were tuned to the lower frequency $|z\rangle \leftrightarrow|-\rangle$ electron spin resonance (see Fig. 1). The full measurement scheme is summarized in Fig. 2(a). 
The fast Fourier transform (FFT) of the electron spin Ramsey oscillation of NV A reveals the spectrum [e.g., Fig. 2(b)] of the $|z\rangle \leftrightarrow|-\rangle$ electron spin transition. The spectrum contains four pairs of spectral lines, where one member of each pair has lower intensity than the other and the splitting $(\approx 70 \mathrm{kHz})$ within these pairs is due to the Stark effect. The amount and splitting of the four pairs originates from hyperfine coupling to the ${ }^{15} \mathrm{~N}$ nuclear spin (refer to Fig. 1), which is described by the addition of the potential [30]

$$
V_{\mathrm{hf}}=A_{\|} S_{z} I_{z}+A_{\perp}\left(S_{x} I_{x}+S_{y} I_{y}\right)
$$

to the spin Hamiltonian (1), where $\vec{I}$ are the $I=1 / 2$ dimensionless nuclear spin operators, $A_{\|}=3.03(3)$ and $A_{\perp}=3.65(3) \mathrm{MHz}$ are the ${ }^{15} \mathrm{~N}$ hyperfine parameters [34]. To second order, the $m_{I}= \pm 1 / 2$ nuclear spin projections

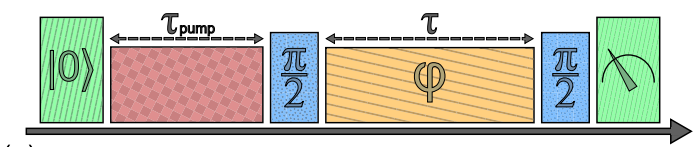

(a)
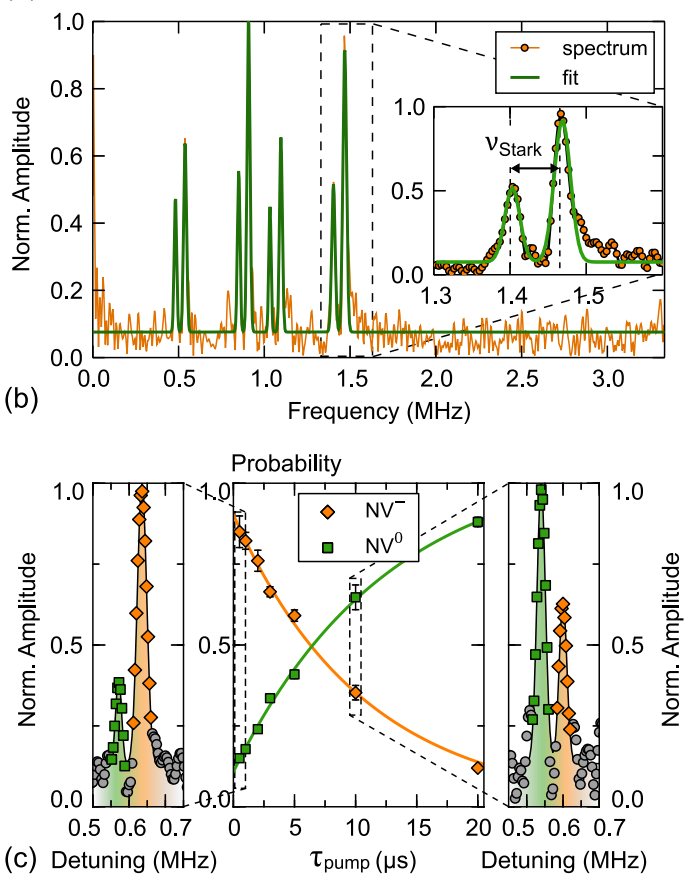

FIG. 2 (color online). (a) Ramsey measurement sequence including charge state preparation of NV $B$ (red laser pulse), spin state initialization and readout of NV $A$ (green laser+ fluorescence detection), microwave pulses for spin control (blue) and free evolution of the spin superposition state (yellow, accumulated phase $\varphi$ during time $\tau$ ). (b) Example Ramsey spectrum revealing four pairs of resonance lines (orange, measurement data, green, multiple Gauss fit). The four pairs are split due to hyperfine interaction (see text). Inset: The splitting within each pair results from the Stark effect. (c) The relative intensity (center) of the Starksplit lines of NV $A$ (left and right inset) can be influenced by pumping NV $B$ from its negative into its neutral charge state with pumping duration $\tau_{\text {pump }}$. Thus demonstrating that the Stark effect originates from different charge states of NV $B$. are degenerate for the $| \pm\rangle$ electron spin states, but are mixed for $|z\rangle$ and split by $\delta f \approx 2 A_{\perp} B_{\perp} / D$ [see Fig. 1(b)]. Consequently, each electron spin resonance becomes two hyperfine lines that are split by $\delta f$. If a small axial magnetic field $B_{z}$ is present, the degeneracy of the nuclear spin projections for the $| \pm\rangle$ electron spin states is lifted and a four-line hyperfine structure becomes observable [see Fig. 2(b)].

The presence of pairs of lines with different intensities in the Ramsey spectrum may be understood by first noting that the spectrum represents a time average of the spin resonances over all measurement runs [31]. Since during some of the measurements $\mathrm{NV} B$ was $\mathrm{NV}^{-}$and during others it was $\mathrm{NV}^{0}$, the Ramsey spectrum contains two sets of hyperfine lines that correspond to the two charge states of NV $B$. The two sets of lines are shifted with respect to each other due to the electric field shift at NV $A$ that results from the change in charge at NV B. Since the electric field shift is smaller than the hyperfine splittings, the two sets of lines appear to form pairs of lines with different intensities. The ratios of the two integrated intensities of the two sets of lines are directly related to the probabilities that $\mathrm{NV} B$ was $\mathrm{NV}^{-}$or $\mathrm{NV}^{0}$ during a measurement. Please note, that ionization of the sensor NV does not affect the measured ratios. To confirm our interpretation, we varied the illumination time $\tau_{\text {pump }}$ with the red charge state, switching lasers in order to vary the charge state probabilities of NV B. Figure 2(c) clearly demonstrates that the ratio of the two sets of lines follows inversely related single exponential curves with $\tau_{\text {pump }}$, which agrees well with the expected variation of the charge state probabilities provided by the current model of the $\mathrm{NV}^{-} \rightarrow \mathrm{NV}^{0}$ photoconversion process $[33,39]$.

Please note that the previously reported spin-spin coupling between the present NV centers [24] does not lead to the observed splitting within the four pairs of resonance lines. This is, first of all, due to the minute coupling of $5 \mathrm{kHz}$, which is smaller than the inhomogeneous linewidth [see Fig. 2(b)] and requires dynamical decoupling sequences to be observed [24]. Second, this minute coupling is even further reduced by our tailoring of the spin eigenstates to be first order insensitive to magnetic fields [15,31].

Given the nontrivial interplay of the magnetic, electric, and hyperfine interactions that govern the observed spin resonances, in order to precisely measure the electric field shift, we recorded the Ramsey spectra for different magnetic field configurations and fit the spectral line frequencies using numerical solutions of the complete spin-Hamiltonian $H+V_{\mathrm{hf}}$. Figure 3(a) depicts the results of a sweep of the auxiliary magnetic field $\delta \vec{B}$, which yields $\Delta f_{-}=66 \pm 7 \mathrm{kHz}$.

Of note for $\mathrm{NV}^{-}$electrometry, the electric field shifts are observed over a much larger range of $B_{z}$ than in the first electrometry demonstration [15], which is due to the larger transverse bias field employed in this work. This outcome promises that $\mathrm{NV}^{-}$electrometry may be successfully implemented in the future with less sophisticated magnetic field alignment. 


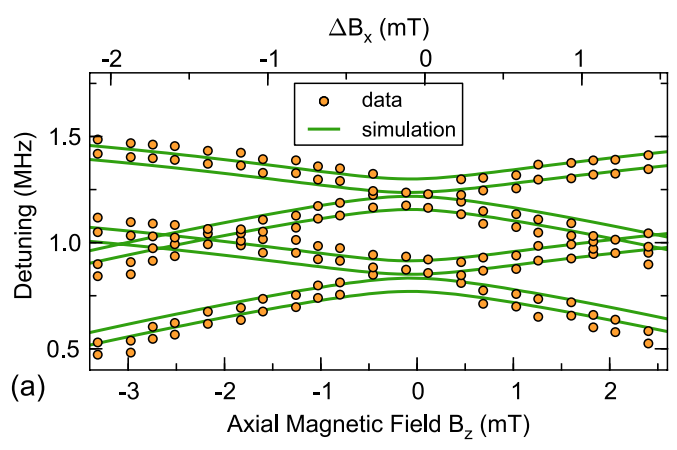

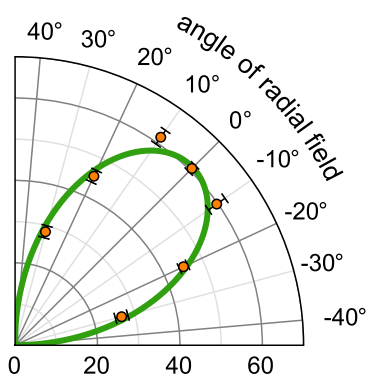

(b) splitting $(\mathrm{kHz})$

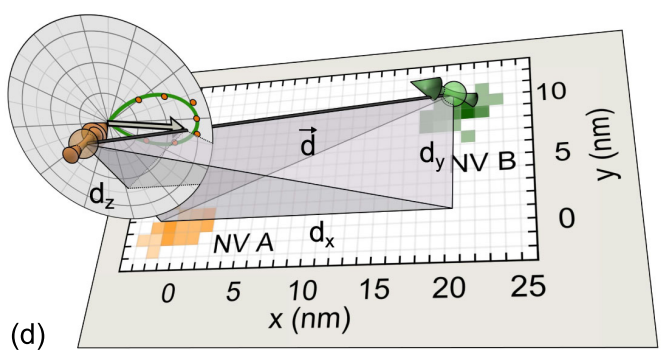

FIG. 3 (color online). (a) Electron spin resonance frequencies as obtained from Ramsey oscillations for different auxiliary magnetic fields $\left(\delta \vec{B}=\Delta B_{x} \hat{x}+B_{z} \hat{z}\right)$. (b) Polar plot of the electric field shift of a single hyperfine line as the transverse magnetic field $B_{\perp}$ is rotated. (c) Superresolution microscopy image of NV $A$ and NV B. (d) Combination of (b),(c) and the known orientations and positions of the centers.

Figure 3(b) depicts the variation of the electric field shift of a single hyperfine line as the transverse magnetic field $B_{\perp}$ was rotated around the $z$ axis. The polar pattern of Fig. 3(b) displays one of the "leaves" of the "four-leaf" pattern predicted by the $\cos \left(2 \phi_{B}+\phi_{E}\right)$ angular dependence of the spin resonances (2) and observed in Ref. [15]. Figure 3(d) combines the ODMR electrometry results with the known positions and orientations of the centers to demonstrate that the polar pattern is orientated towards NV $B$ from NV $A$. Given the expected angular dependence, this suggests that the transverse electric field at NV $A$ is similarly orientated. Figure 3(d) also demonstrates that the displacement vector $\vec{d}$ connecting NV $A$ and NV B. Electric field simulation yields a field angle of $6 \pm 4^{\circ}$ and and $\Delta f_{\text {sim }}=70 \pm 10 \mathrm{kHz}$. Considering the orientation of an electric field at NV $A$ generated by a charge at NV $B$, this angle and the observed net electric field shift of $\Delta f_{-} \approx$ $66 \mathrm{kHz}$ implies that the shift due to the axial component of the electric field is $k_{\|} E_{z}<0.3 \mathrm{kHz}$, which is too small to be detected. Specifying $k_{\|} E_{z} \sim 0$, the fit of the magnetic field data of Fig. 3(a) yields the transverse electric field shift $k_{\perp} E_{\perp}=66 \pm 7 \mathrm{kHz}$ and a field angle of $0.5 \pm 4^{\circ}$ which is in good agreement with the calculated values from the distance vector.

Accounting for the relative permittivity of diamond $\left(\epsilon_{r}=5.7\right)$, the measured transverse electric field is that of a single electron located at a transverse distance of $25 \pm 2 \mathrm{~nm}$ from NV $A$, which is consistent with the superresolution microscopy measurement of $27 \pm 3 \mathrm{~nm}$. Noting that the measured electric field is the difference in the electric field at NV $A$ due to the change of charge at $\mathrm{NV} B$, this result may be interpreted as the displacement of the excess electron at $\mathrm{NV} B$ when it is $\mathrm{NV}^{-}$to a position beyond the range of detection $(>40 \mathrm{~nm})$.

Combining our evidence obtained from optically controlling the NV charge state, observing the OMDR as a function of magnetic field strength and direction and interpreting superresolution microscopy, it is clear that we successfully employed one NV center as an electrometer to detect the single excess electron that determines the charge state of another NV center located $\approx 25 \mathrm{~nm}$ away. Thus demonstrating single charge detection using $\mathrm{NV}^{-}$ electrometry under ambient conditions and unequivocally confirming the current charge state assignments. Furthermore, our measurements provided direct insight into the microscopic behavior of the excess electron. Combined with recent advances in nanoscale NV center metrology $[19,20,23]$ and in artificial NV center creation yielding long coherence times even close to diamond surfaces [49], the knowledge gained by this proof of principle experiment will lead to nanoscale metrology scenarios. In addition, single shot readout of NV spins [50,51] could be applied for single shot charge detection, given a deterministic correlation between the NV spin state and the presence or absence of a charge as in the present case.

The authors would like to acknowledge financial support by the ARC (DP120102232); the EU via projects SQUTEC, DIADEMS, and Diamant; the DFG via the SFB/TR21, the research groups 1493 "Diamond quantum materials" and 1482; the Volkswagen Foundation; the BMBF via programs QuORep, Q.Com, and ChistEra; and the Max Planck Society.

*f.dolde@physik.uni-stuttgart.de

[1] M. J. Yoo, T. A. Fulton, H. F. Hess, R. L. Willett, L. N. Dunkleberger, R. J. Chichester, L. N. Pfeiffer, and K. W. West, Science 276, 579 (1997).

[2] J. Martin, N. Akerman, G. Ulbricht, T. Lohmann, J. H. Smet, K. von Klitzing, and A. Yacoby, Nat. Phys. 4, 144 (2008).

[3] C. Schonenberger and S. F. Alvarado, Phys. Rev. Lett. 65, 3162 (1990).

[4] M. H. Devoret and R. J. Schoelkopf, Nature (London) 406, 1039 (2000).

[5] J. M. Elzerman, R. Hanson, L. H. Willems van Beveren, B. Witkamp, L. M. K. Vandersypen, and L. P. Kouwenhoven, Nature (London) 430, 431 (2004). 
[6] F. Patolsky, G. Zheng, and C. M. Lieber, Nat. Protoc. 11711 (2006).

[7] Y. Cui, Q. Wei, H. Park, and C. M. Lieber, Science 293, 1289 (2001).

[8] C. C. Williams, J. Slinkman, W.P. Hough, and H. K. Wickramasinghe, Appl. Phys. Lett. 551662 (1989).

[9] A. K. Henning, T. Hochwitz, J. Slinkman, J. Never, S. Hoffmann, P. Kaszuba, and C. Daghlian, J. Appl. Phys. 77, 1888 (1995).

[10] Y. Martin, D. W. Abraham, and H. K. Wickramasignhe, Appl. Phys. Lett. 52, 1103 (1988).

[11] A. N. Cleland and M. L. Roukes, Nature (London) 392, 160 (1998).

[12] J. S. Bunch, A. M. van der Zande, S. S. Verbridge, I. W. Frank, D. M. Tanenbaum, J. M. Parpia, H. G. Craighead, and P. L. McEuen, Science 315, 490 (2007).

[13] J. Salfi, I. G. Savelyev, M. Blumin, S. V. Nair, and H. E. Ruda, Nat. Nanotechnol. 5, 737 (2010).

[14] J. Lee, Y. Zhu, and A. Seshia, J. Micromech. Microeng. 18, 025033 (2008).

[15] F. Dolde, H. Fedder, M. W. Doherty, T. Nöbauer, F. Rempp, G. Balasubramanian, T. Wolf, F. Reinhard, L. C. L. Hollenberg, F. Jelezko, and J. Wrachtrup, Nat. Phys. 7, 459 (2011).

[16] G. Balasubramanian., I. Y. Chan, R. Kolesov, M. Al-Hmoud, J. Tisler, C. Shin, C. Kim, A. Wojcik, P. R. Hemmer, A. Krueger, T. Hanke, A. Leitenstorfer, R. Bratschitsch, F. Jelezko, and J. Wrachtrup, Nature (London) 455, 648 (2008).

[17] M. S. Grinolds, S. Hong, P. Maletinsky, L. Luan, M. D. Lukin, R. L. Walsworth, and A. Yacoby, Nat. Phys. 9, 215 (2013).

[18] G. Waldherr., J. Beck, P. Neumann, R. S. Said, M. Nitsche, M. L. Markham, D. J. Twitchen, J. Twamley, F. Jelezko, and J. Wrachtrup, Nat. Nanotechnol. 7, 105 (2012).

[19] T. Staudacher, F. Shi, S. Pezzagna, J. Meijer, J. Du, C. A. Meriles, F. Reinhard, and J. Wrachtrup, Science 339, 561 (2013).

[20] H. J. Mamin, M. Kim, M. H. Sherwood, C. T. Rettner, K. Ohno, D. D. Awschalom, and D. Rugar, Science 339, 557 (2013).

[21] D. M. Toyli, C. F. de las Casas, D. J. Christle, V. V. Dobrovitski, and D. D. Awschalom, Proc. Natl. Acad. Sci. U.S.A. 110, 8417 (2013).

[22] P. Neumann, I. Jakobi, F. Dolde, C. Burk, R. Reuter, G. Waldherr, J. Honert, T. Wolf, A. Brunner, J. H. Shim, D. Suter, H. Sumiya, J. Isoya, and J. Wrachtrup, Nano Lett. 13, 2738 (2013).

[23] G. Kucsko, P. C. Maurer, N. Y. Yao, M. Kubo, H. J. Noh, P. K. Lo, H. Park, and M. D. Lukin, Nature (London) 500, 54 (2013).

[24] F. Dolde, I. Jakobi, B. Naydenov, N. Zhao, S. Pezzagna, C. Trautman, J. Meijer, P. Neumann, F. Jelezko, and J. Wrachtrup, Nat. Phys. 9, 139 (2013).

[25] H. Bernien, B. Hensen, W. Pfaff, G. Koolstra, M. S. Blok, L. Robledo, T. H. Taminiau, M. Markham, D. J. Twitchen, L. Childress, and R. Hanson, Nature (London) 497, 86 (2013).

[26] L. P. McGuinness, Y. Yan, A. Stacey, D. A. Simpson, L. T. Hall, D. Maclaurin, S. Prawer, P. Mulvaney, J. Wrachtrup, F. Caruso, R. E. Scholten, and L. C. L. Hollenberg, Nat. Nanotechnol. 6, 358 (2011).

[27] D. M. Toyli, D. J. Christle, A. Alkauskas, B. B. Buckley, C. G. Van de Walle, and D. D. Awschalom, Phys. Rev. X 2, 031001 (2012).

[28] M. W. Doherty, V. V. Struzhkin, D. A. Simpson, L. P. McGuinness, Y. Meng, A. Stacey, T. J. Karle, R. J. Hemley,
N. B. Manson, L. C. L. Hollenberg, and S. Prawer, [Phys. Rev. Lett. (to be published)].

[29] A. Gruber, A. Drabenstedt, C. Tietz, L. Fleury, J. Wrachtrup, and C. von Borczyskowski, Science 276, 2012 (1997).

[30] M. W. Doherty, N. B. Manson, P. Delaney, F. Jelezko, and L. C. L. Hollenberg, Phys. Rep. 528, 1 (2013).

[31] M. W. Doherty, F. Dolde, H. Fedder, F. Jelezko, J. Wrachtrup, N. B. Manson, and L. C. L. Hollenberg, Phys. Rev. B 85, 205203 (2012).

[32] E. van Oort and M. Glasbeek, Chem. Phys. Lett. 168, 529 (1990).

[33] See Supplemental Material at http://link.aps.org/ supplemental/10.1103/PhysRevLett.112.097603 for experimental and theoretical details.

[34] S. Felton, A. M. Edmonds, M. E. Newton, P. M. Martineau, D. Fisher, D. J. Twitchen, and J. M. Baker, Phys. Rev. B 79, 075203 (2009).

[35] J. H. N. Loubser and J. A. van Wyk, Rep. Prog. Phys. 41, 1201 (1978).

[36] M. V. Hauf, B. Grotz, B. Naydenov, M. Dankerl, S. Pezzagna, J. Meijer, F. Jelezko, J. Wrachtrup, M. Stutzmann, F. Reinhard, and J. A. Garrido, Phys. Rev. B 83, 081304(R) (2011).

[37] B. Grotz., M. V. Hauf, M. Dankerl, B. Naydenov, S. Pezzagna, J. Meijer, F. Jelezko, J. Wrachtrup, M. Stutzmann, F. Reinhard, and J. A. Garrido, Nat. Commun. 3, 729 (2012).

[38] K. Y. Han, S. K. Kim, C. Eggeling, and S. W. Hell, Nano Lett. 10, 3199 (2010).

[39] G. Waldherr, J. Beck, M. Steiner, P. Neumann, A. Gali, Th. Frauenheim, F. Jelezko, and J. Wrachtrup, Phys. Rev. Lett. 106, 157601 (2011).

[40] K. Beha, A. Batalov, N. B. Manson, R. Bratschitsch, and A. Leitenstorfer, Phys. Rev. Lett. 109, 097404 (2012).

[41] K. Y. Han, D. Wildanger, E. Rittweger, J. Meijer, S. Pezzagna, S. W. Hell, and C. Eggeling, New J. Phys. 14, 123002 (2012).

[42] N. Aslam, G. Waldherr, P. Neumann, F. Jelezko, and J. Wrachtrup, New J. Phys. 15, 013064 (2013).

[43] N. B. Manson and J. P. Harrison, Diam. Relat. Mater. 14, 1705 (2005).

[44] Y. Mita, Phys. Rev. B 53, 11360 (1996).

[45] A. Gali, Phys. Rev. B 79, 235210 (2009).

[46] J. R. Weber, W. F. Koehl, J. B. Varley, A. Janotti, B. B. Buckley, C. G. Van de Walle, and D. D. Awschalom, Proc. Natl. Acad. Sci. U.S.A. 107, 8513 (2010).

[47] A. Ranjbar, M. Babamoradi, M. Heidari Saani, M. A. Vesaghi, K. Esfarjani, and Y. Kawazoe, Phys. Rev. B 84, 165212 (2011).

[48] S. Pezzagna, D. Rogalla, H.-W. Becker, I. Jakobi, F. Dolde, B. Naydenov, J. Wrachtrup, F. Jelezko, C. Trautmann, and J. Meijer, Phys. Status Solidi A 208, 2017 (2011).

[49] T. Yamamoto, C. Müller, L. P. McGuinness, T. Teraji, B. Naydenov, S. Onoda, T. Ohshima, J. Wrachtrup, F. Jelezko, and J. Isoya, Phys. Rev. B 88, 201201 (2013).

[50] P. C. Maurer, G. Kucsko, C. Latta, L. Jiang, N. Y. Yao, S. D. Bennett, F. Pastawski, D. Hunger, N. Chisholm, M. Markham, D. J. Twitchen, J. I. Cirac, and M. D. Lukin, Science 336, 1283 (2012).

[51] P. Neumann, J. Beck, M. Steiner, F. Rempp, H. Fedder, P. R. Hemmer, J. Wrachtrup, and F. Jelezko, Science 329, 542 (2010). 